\title{
CONDICIONANTES ECONÓMICAS DE COLOMBIA Y LA ALIANZA DEL PACÍFICO
}

\author{
DETECTING ECONOMIC CONDITIONS FOR COLOMBIA ANDTHE PACIFIC ALLIANCE
}

\section{DETERMINAÇÃO AS CONDIÇÕES ECONÔMICAS DA COLÔMBIA E DA ALIANÇA DO PACÍFICO}

\author{
Por: REYES - Giovanni E., MEDINA GARCÍA - Mayerly
}

\begin{abstract}
Ph.D. en Economía para el Desarrollo y Relaciones Internacionales de la Universidad de Pittsburgh, con certificados de post-grado de las Universidades de Pennsylvania y Harvard. Profesor Titular de la Universidad Colegio Mayor de Nuestra Señora del Rosario, Bogotá, Colombia.

Profesora de Carrera, Especialista en Relaciones Industriales y Gerencia de Recursos Humanos, es Directora de Bienestar y Gestión Humana en la Corporación Universitaria Minuto de Dios, Bogotá, Colombia.
\end{abstract}

\section{RESUMEN}

La principal finalidad de este estudio es presentar una visión actualizada y genérica respecto a las condicionantes económicas de Colombia en particular y las relacionadas con el conjunto de países que conforman la Alianza del Pacífico en general -Chile, Colombia, México y Perú. Se trata de un estudio no experimental, descriptivo-interpretativo con énfasis en la revisión de datos. Entre las conclusiones más importantes se destacan los niveles de crecimiento económico con mayor estabilidad que presentan Chile y Perú, una dependencia comercial exterior de México hacia Estados Unidos y en Colombia se tiene el caso de que el aumento de producción que se hace evidente no impacta tanto como era de esperarse, en la variable empleo.

Palabras clave: Alianza del Pacífico, economía latinoamericana; economía de Colombia.

JEL: E10, E20, E66.

\section{ABSTRACT}

The main aim of this study is to present a general overview concerning the current economic conditions of the Colombian economy and the group of countries 
that form the Pacific Alliance, namely: Chile, Colombia, Mexico and Peru. This is a non-experimental descriptive-interpretative study with particular emphasis on data review. Among the most important findings it is possible to highlight levels of a more stable economic growth that is evident in Chile and Peru, an external trade dependence regarding the relationship between Mexico and the United States and acceptable economic growth levels in the Colombian economy, even though this increase in production is not showing important levels of impact on employment conditions within domestic market.

Key words: Pacific Alliance, Latin American economy, Colombian economy.

JEL: E10, E20, E66.

\section{RESUMO}

A principal finalidade deste estudo é apresentar uma visão atualizada e geral sobre as condições econômicas da Colômbia em particular e as relacionadas com o conjunto de países que conformam a Aliança do Pacífico, em geral - Chile, Colômbia, México e Peru. Trata-se de um estudo não experimental, descritivo e interpretativo com ênfases na revisão de dados. Entre as conclusões mais importantes se destacam os níveis de crescimento econômico com maior estabilidade e que se presentam nos países de Chile e Peru, uma dependência comercial exterior do México para Estados Unidos e que na Colômbia há o caso do evidente aumento de produção e que não impacta tanto como era de se esperar, na variável de emprego.

Palavras chaves: Aliança do Paćíico, economia Latino-americana; economia da Colômbia.

JEL: E10, E20, E66. 


\section{INTRODUCCIÓN}

El objetivo fundamental de este documento es establecer una caracterización socio-económica de Colombia y de los países que conforman la Alianza del Pacífico. Colombia ha desempeñado un papel destacado en esta Alianza; no obstante, el Presidente Alan García de Perú, fue quien llevó a cabo la primera convocatoria de este grupo de integración regional. En efecto, este mandatario el 28 de abril de 2011, convocó a una reunión a sus homólogos de Chile, Colombia y México

Producto de la citada reunión fue firmado el documento "Declaración de Lima”. Este documento establece que el propósito fundamental de la Alianza es "profundizar la integración entre estas economías y definir acciones conjuntas de Chile, Colombia, México y Panamá para la vinculación comercial con los países asiáticos de la Cuenca del Pacífico, sobre la base de acuerdos comerciales bilaterales existentes entre los Estados parte”.

En los últimos siete años, en especial luego de la última crisis financiera internacional que tuvo su punto de inflexión el 13 de septiembre de 2008, con la quiebra del banco de inversión Lehmann Brothers, los países de la Alianza han tenido crecimiento económico notable en América Latina. El mismo ha sido superior al crecimiento del producto interno bruto (PIB) de otro bloque que opera en Suramérica, el Mercado Común del Sur (MERCOSUR) el que se encuentra integrado por Argentina, Brasil, Paraguay, Uruguay y Venezuela ${ }^{2}$.

A los países integrantes de la Alianza del Pacífico se les ha identificado como los “pumas de América Latina”, en comparación con los "tigres asiáticos” (Corea del Sur, Hong Kong, Singapur y Taiwan). Otro aspecto importante de la Declaración de Lima, establece que la intención del grupo de países es "alentar la integración regional así como un mayor crecimiento, desarrollo y competitividad de los países miembro".

La Alianza tiende a sobreponerse en alcances y actualidad a la Comunidad Andina (CAN) un grupo de países cuyos esfuerzos de integración datan de 1969 con la firma del Acuerdo de Cartagena. En un inicio este bloque promovió la integración como un medio para el desarrollo de los países, esencialmente utilizando la desviación del comercio, lo que implicó la protección de las industrias locales. La CAN ha tenido las críticas enfocadas a señalar una gran dimensión de su institucionalidad, situación que la colocaría como burocrática. En este sentido, el Mercado Común del Sur (MERCOSUR) parece ser una instancia de integración más flexible.

1. Para mayores referencias, véase el enlace: https:/alianzapacifico.net/que-es-la-alianza/\#antecedentes, (consultado por última vez, el 26 de junio de 2016). Véase además: 2015) Negocios Proméxico: The Pacific Alliance Issue. (México, D.F.: Proméxico) especialmente pps, 54-60, 71-76.

2. Mayor discusión sobre las condicionantes de integración latinoamericana y la búsqueda de nuevos mercados, especialmente en Asia, véase: Kuwayama, Mikio (2005) Hacia una Nueva Alianza de Comercio e Inversión entre América Latina y Asia-Pacífico (Washington, D.C.: Banco Interamericano de Desarrollo, Instituto para la Integración de América Latina y el Caribe, BID-INTAL) en especial pp. 10-23, 28-31. 
Por su parte, la Alianza del Pacífico tiene una mínima institucionalidad y depende básicamente de la presidencia temporal, que la ejercen rotativamente los integrantes de la Alianza. Las acciones que desde allí se emprenden, son coordinadas a través de los ministerios y las agencias de los cuatros países integrantes, lo cual permite entender, que no existe un sede única desde donde se sesione. Existe un Grupo de Alto Nivel GAN-, que se encarga de validar y garantizar que los objetivos y las decisiones que se adopten estén sujetos al Acuerdo Marco y a lo contenido en las declaraciones presidenciales de la AP, son quienes realizan seguimiento y supervisión así como los que establecen contacto con otros organismos o grupos externos.

A continuación, los diferentes apartados abordan temas económicos fundamentales en el desempeño de los países que hacen parte de la Alianza y se incluyen aspectos relacionados con la ampliación del mercado interno, condicionantes de mercados internacionales y de intercambios comerciales dentro del bloque.

\section{ALIANZA DEL PACÍFICO: FINALIDADES PRINCIPALES}

De conformidad con información actualizada de documentos oficiales (véase el enlace: https://alianzapacifico.net/) la Alianza del Pacífico (AP) se creó el 28 de abril de 2011 y se estableció formalmente el 6 de junio de 2012. Con base en la producción total anual de sus cuatro miembros (Chile, Colombia, México y Perú) constituye la octava economía y la octava potencia exportadora en el mundo.

Sus principales finalidades, de conformidad con el texto del Acuerdo Marco, se enfocan a:

- La completa liberación del comercio intrarregional. Con el fin de lograr esta finalidad, se presentó un plan consistente en: (i) la desgravación inmediata del 92 por ciento de los aranceles; (ii) el 8 por ciento que queda de remanente se logrará en plazos que van de 3 a 7 años; y complementariamente, (iii) un grupo pequeño de productos que se estima más estratégico o sensitivo, tendrá reducción de sus aranceles en 10 años.

- Fomento a la integración productiva. Con el fin de promover la producción, el empleo y la competitividad interna del bloque, los exportadores de los países considerarán como nacionales los insumos comprados a sus socios; con ello lo que se busca, adicionalmente, es el fomento al comercio intrarregional y la integración productiva complementaria.

- Movilidad de ciudadanos. Los países han acordado eliminar la totalidad de visas para turistas entre los países miembros de la AP; de manera más actualizada, comisiones técnicas trabajan en el diseño de mecanismos que puedan facilitar el flujo de personas en la región.

- Fomento conjunto al comercio e inversión. Las respectivas instituciones encargadas de la promoción en los países de la AP, han acordado una serie de actividades, tendientes a promover tanto el comercio como la inversión de los países miembros coadyuvando con ello a la constitución integrada de un bloque productivo. 
- Intercambio de buenas prácticas en áreas de interés. Las áreas que se incluyen aquí son las referentes al cambio climático y el fomento de pequeñas y medianas empresas entre los países miembros.

- Constitución del Fondo de Cooperación. Se constituye un fondo con los aportes de los países miembros; la finalidad del mismo es el financiamiento de los actuales y futuros proyectos. La temática a abordar incluye el medio ambiente, desarrollo tecnológico y sostenibilidad empresarial a largo plazo.

\section{COLOMBIA Y PAIISES LATINOAMERICANOS EN 2015: VARIABLES ECONÓMICAS}

Considerada de manera conjunta la capacidad de producción de los cuatro países que conforman la AP, se constituirían en la octava economía del mundo y en el bloque comercial que ocuparía la posición número ocho en el ámbito mundial con potencial exportador, como se demuestra en la tabla 1. Las 7 economías más grandes son: China, Estados Unidos, Japón, India, Francia, Reino Unido e Italia. Conforme a esta evidencia, es notable advertir la destacada capacidad del mercado interno del bloque, toda vez que sobrepasa los 200 millones de habitantes.

Tabla 1. Alianza del Pacífico 2015: Caracterización general de PIB, población y PIBpc

\begin{tabular}{lccc}
\hline País & $\begin{array}{c}\text { Producto Interno } \\
\text { Bruto } \\
\text { (miles de millones } \\
\text { de US\$) }\end{array}$ & $\begin{array}{c}\text { Población } \\
\text { (millones de } \\
\text { habitantes) }\end{array}$ & $\begin{array}{c}\text { PIB per cápita } \\
\text { (poder paritario } \\
\text { de compra, ppp en } \\
\text { US\$) }\end{array}$ \\
\hline Chile & 268 & 17 & 18419 \\
\hline Colombia & 366 & 47 & 10792 \\
\hline México & 1177 & 115 & 15312 \\
\hline Perú & 199 & 30 & 10719 \\
\hline
\end{tabular}

Fuente: Comisión Económica para América Latina y el Caribe (CEPAL) (2015) Balance Preliminar de las Economías de América Latina y el Caribe 2015. (Santiago de Chile, Chile: CEPAL); y estadísticas del Fondo Monetario Internacional (véase: http://www.imf. org/external/index.htm).

Es decir que, además de la competitividad que puede desarrollar para insertarse en los mercados externos, tiene un significativo potencial para que las economías crezcan y se fortalezcan en su competitividad, dada la demanda agregada interna de los países. Nótese que dentro de la capacidad de demanda, y teniendo en cuenta el poder paritario de compra, aunque es el país menos poblado, Chile tiene el mayor poder adquisitivo. No obstante, México que contiene a más de la mitad del total de población de la AP tiene el segundo lugar en capacidad de compra en los mercados internos.

En ese mismo orden de ideas, Colombia se encuentra con una capacidad de compra, determinada por el PIB per cápita similar a la que se evidencia en Perú. 
De hecho una de las mayores críticas que se hacen al modelo de crecimiento económico de estos países es que no han podido resolver tanto como hubiese sido deseable, los altos niveles de inequidad en el ingreso. Situación que por otra parte también es atribuible a Chile y a México.

\section{COLOMBIA Y PAÍSES LATINOAMERICANOS EN 2015: ÍNDICE DE MISERIA Y DESEMPEÑO ECONÓMICO}

Con base en las más recientes cifras disponibles respecto a los países de América Latina, correspondientes a 2015, se realizó el presente estudio que tiene por objeto determinar el ambiente de negocios prevaleciente en la región. Los indicadores generales que se presentan son dos: (i) el índice de la miseria, que es igual a: la desocupación total más la inflación, considerada esta última como elevación generalizada de precios en un país; y (ii) el índice de desempeño económico, que es igual a: crecimiento económico dividido entre la suma de inflación más desempleo. Es decir qué tanta cobertura tiene el aumento de la producción en relación con el índice de miseria (CEPAL, 2015).

El índice de miseria, está relacionado con dos indicadores que son adversos a la estabilidad y al crecimiento de una economía. Son datos que se vinculan por lo general con un contraproducente clima de negocios y que muchas veces se retroalimentan de manera mutua: a más inflación más inseguridades para muchas inversiones, lo que afecta negativamente la creación de puestos de trabajo.

Mientras más alto es el índice de miseria, mayor es la probabilidad de que el país en estudio, se encuentre en condiciones de estanflación. Esto es, con altos índices de paro y con significativos valores en la elevación de precios de bienes y servicios, lo que hace contraer de manera drástica la capacidad adquisitiva de las personas y con ello la demanda efectiva de los mercados internos.

En el segundo caso, el índice de desempeño aglutina en un solo indicador, la presencia de tres variables -inflación, desempleo y crecimiento económico- que son vitales para conocer de manera rápida, la situación del mercado interno de un país.

La tabla 2 muestra los índices de miseria. De acuerdo a este indicador agregado, la región latinoamericana evidencia haber superado las condiciones de hiperinflación que caracterizaron a los años ochenta. Las cifras del desempleo abierto son oficiales y en mucho de los países de la región, es preciso enfatizar que el problema laboral más que de desocupación total reside en el sub-empleo, es decir en actividades que se encuentran en la informalidad o en los circuitos de la economía subterránea.

Esto se traduce -junto a otros factores-y en particular cuando las condiciones son dramáticas, en notables inestabilidades socio-políticas, tal el caso de lo que se presenta actualmente en Venezuela y Brasil.

Son nueve los países que aparecen teniendo bastante bajo control el índice de miseria en ese orden: Panamá, Guatemala, El Salvador, México, República Dominicana, Trinidad \& Tobago, Costa Rica, Ecuador y Nicaragua. En varios de estos casos, las remesas ocupan un lugar importante. Siendo estas las condicionantes 
que incluyen en especial a Guatemala, El Salvador, Nicaragua y hasta cierto punto México y República Dominicana.

Tabla 2. Índice de miseria en América Latina y el Caribe $2015^{1}$

\begin{tabular}{|c|c|c|c|}
\hline País & $\begin{array}{c}\text { Índice de Miseria } \\
\text { (inflación }+ \\
\text { desempleo) }\end{array}$ & Grupo & $\begin{array}{c}\text { Promedio de } \\
\text { Grupo }\end{array}$ \\
\hline Panamá & 4.1 & \multirow{3}{*}{ Bajo } & \multirow{3}{*}{5.76} \\
\hline Guatemala & 6.5 & & \\
\hline El Salvador & 6.7 & & \\
\hline México & 7.6 & \multirow{9}{*}{ Moderado Bajo } & \multirow{9}{*}{9.06} \\
\hline República Dominicana & 7.7 & & \\
\hline Trinidad \& Tobago & 8.5 & & \\
\hline Costa Rica & 8.7 & & \\
\hline Ecuador & 8.8 & & \\
\hline Nicaragua & 9.7 & & \\
\hline Bolivia & 10.1 & & \\
\hline Perú & 10.2 & & \\
\hline Chile & 10.3 & & \\
\hline Paraguay & 10.4 & \multirow{3}{*}{ Intermedio } & \multirow{3}{*}{11.60} \\
\hline Honduras & 11.3 & & \\
\hline Jamaica & 13.1 & & \\
\hline Colombia & 15.5 & \multirow{3}{*}{ Moderado Alto } & \multirow{3}{*}{16.03} \\
\hline América Latina y el Caribe & 15.8 & & \\
\hline Brasil & 16.8 & & \\
\hline Uruguay & 17.1 & \multirow{2}{*}{ Alto } & \multirow{2}{*}{18.85} \\
\hline Argentina & 20.6 & & \\
\hline Venezuela & 79.8 & Peligrosamente Alto & 79.8 \\
\hline
\end{tabular}

Notas: $\quad 1 /$ Índice de Miseria: IM = [tasa anual de inflación + tasa anual de desempleo abierto)]. El análisis por agrupaciones ("clusters") se basó en un rango de 16.5 -excluyéndose el valor extremo de Venezuela que distorsionaba la serie regional. El intervalo de cada grupo de quintiles fue de 3.3 .

Fuente: Cálculos del estudio, con base en series históricas de FMI, CEPAL, BID y Banco Mundial; estas series se han calculado sobre la base de US\$ de 2010.

Trinidad \& Tobago se ha visto afectada favorablemente por la situación petrolera que aún mantenía elevados niveles para principios de 2015 y por el turismo. En el caso de Nicaragua y Ecuador, las políticas de expansión fiscal han jugado un notable papel, generando oportunidades laborales. 
Tabla 3. Índice de desempeño económico en los países de América Latina y el Caribe 2015 $\left(\mathrm{IDE}=\left[(\text { tasa de crecimiento económico) } /(\text { inflación }+ \text { desempleo })]^{*} 100\right)\right.$

\begin{tabular}{|c|c|c|c|}
\hline País & $\begin{array}{l}\text { Índice de } \\
\text { Desempeño } \\
\text { Económico }\end{array}$ & Grupo & $\begin{array}{c}\text { Promedio de } \\
\text { Grupo }\end{array}$ \\
\hline Panamá & 143.90 & Alto & 143.90 \\
\hline República Dominicana & 85.71 & Moderado Alto & 85.71 \\
\hline Guatemala & 60.00 & Intermedio & 60.00 \\
\hline Bolivia & 44.55 & \multirow{5}{*}{ Moderado Bajo } & \multirow{5}{*}{37.31} \\
\hline Nicaragua & 42.27 & & \\
\hline El Salvador & 35.82 & & \\
\hline México & 32.89 & & \\
\hline Costa Rica & 31.03 & & \\
\hline Honduras & 30.09 & \multirow{11}{*}{ Bajo } & \multirow{11}{*}{14.58} \\
\hline Paraguay & 27.88 & & \\
\hline Perú & 27.45 & & \\
\hline Colombia & 20.00 & & \\
\hline Chile & 19.42 & & \\
\hline Argentina & 9.71 & & \\
\hline Uruguay & 8.77 & & \\
\hline Jamaica & 7.63 & & \\
\hline Ecuador & 4.55 & & \\
\hline América Latina y el Caribe & 2.53 & & \\
\hline Trinidad \& Tobago & 2.35 & & \\
\hline Venezuela & -8.90 & \multirow{2}{*}{ Peligrosamente Bajo } & \multirow{2}{*}{-14.87} \\
\hline Brasil & -20.83 & & \\
\hline
\end{tabular}

Notas: El análisis por agrupaciones ("clusters") se basó en un rango de 141.5 -excluyéndose los valores de Venezuela y Brasil que siendo negativos distorsionaban la serie regional. El intervalo de cada grupo de quintiles fue de 28.3.

Fuente: |Cálculos del estudio, con base en series históricas de FMI, CEPAL, BID y Banco Mundial; estas series se han calculado sobre la base de US\$ de 2010.

En el otro extremo, Argentina y Venezuela tienen niveles alarmantes en cuanto a la combinación de inflación y desempleo. Pero los retos son dramáticamente más importantes para el país que se conduce desde Caracas. El valor extremo de índice de la miseria de Venezuela hace que el análisis regional deba realizarse incluyendo a Venezuela y luego excluyendo a este país, dado que los indicadores -especialmente promedios o medidas de tendencia central- se ven muy influenciados por los extremos. 
Venezuela tiene una combinación de inflación y desempleo que es 20 veces la que corresponde a Panamá, quien es el país con el índice más favorable en este sentido. Es tal la condición venezolana que puede estar al borde de una crisis humanitaria, dada la escasez de alimentos y bienes que cubren necesidades básicas.

En cuanto al desempeño económico -Cuadro 3- el caso más extraordinario y favorable en la región es Panamá. Con un indicador de 143 por ciento, se tiene que el crecimiento económico, que es el más alto en América Latina, supera a la combinación de inflación y desempleo. Nótese cómo este valor extremo jalona toda el conjunto de datos, de manera que en los grupos de moderado alto e intermedio, se tiene solamente un país en cada uno de esos apartados (Reyes, 2007).

En algunos casos, el índice de miseria estaba muy controlado, pero el crecimiento económico no fue notablemente alto, de manera que el índice de desempeño no adquirió los altos niveles que podrían haberse esperado. Este sería el caso de El Salvador y Costa Rica. En otros países, el crecimiento económico tuvo un comportamiento negativo y de allí que el desempeño sea peligrosamente bajo, tal el caso de Brasil.

Los desempeños económicos bajos se asocian con condicionantes de inestabilidad en la relación de los grupos sociales y en dificultades de legitimidad de las instituciones públicas. De nuevo los escenarios actuales de Brasil y de Venezuela son prueba de ello.

En el caso de Colombia, el índice de miseria es moderado alto y consecuentemente, cumpliendo con la tendencia que es de es esperarse en estos casos, el índice de desempeño es bajo. Esta última condición la comparte Colombia con otros países de la región especialmente Chile, Argentina, Ecuador y el promedio de toda la región (ONU, 2006).

De manera complementaria a los dos estudios generales de ambos indicadores en toda la región, se realizó un análisis en función de: (i) sub-regiones de Latinoamérica y el Caribe; y (ii) tamaños de las economías.

Tabla 4. Subregiones de América Latina y el caribe 2015: promedios y desviaciones estándar de índice de miseria y de desempeño económico

\begin{tabular}{lcccc}
\hline \multicolumn{1}{c}{ Subregión } & \multicolumn{2}{c}{ Índice de Miseria } & \multicolumn{2}{c}{$\begin{array}{c}\text { Índice de Desempeño } \\
\text { Económico }\end{array}$} \\
& Promedio & $\begin{array}{c}\text { Desviación } \\
\text { Estándar }\end{array}$ & Promedio & $\begin{array}{c}\text { Desviación } \\
\text { Estándar }\end{array}$ \\
\hline México-Centroamérica-Caribe & 8.39 & 2.55 & 47.17 & 41.48 \\
Andinos & 24.88 & 30.81 & 17.53 & 16.62 \\
\hline Mercosur + Chile & 15.04 & 4.53 & 8.22 & 9.02 \\
\hline
\end{tabular}

Notas: (i) México-Centroamérica-Caribe: Costa Rica, El Salvador, Guatemala, Honduras, Jamaica, México, Nicaragua, Panamá, República Dominicana y Trinidad \& Tobago; (ii) Andinos: Bolivia, Colombia, Ecuador, Perú y Venezuela; y (iii) Mercosur+Chile: Argentina, Brasil, Chile, Paraguay y Uruguay.

Fuente: Cálculos del estudio, con base en Tablas 1 y 2. 
En la tabla 4, se muestran los resultados de las subregiones y según los datos, tanto el mejor desempeño económico como el más bajo nivel en el índice de miseria, se tienen en México-Centroamérica y Caribe o sub región mesoamericana.

Para el promedio en los dos indicadores agregados en esta sub-región, influye notablemente el hecho de que Panamá es significativamente un país con mejores condiciones macroeconómicas en sus características vitales de empleo, crecimiento de producción y estabilidad de precios.

Para este país, el factor de los sectores financieros tiene una importante repercusión, además del sector de la construcción, que en los últimos años se vio fortalecido por las obras de ampliación del Canal. Los resultados son contrastantes al comparar las subregiones de MERCOSUR+Chile -o Cono Sur- y la de los países Andinos.

Los índices de miseria son más bajos en el Cono Sur, mientras que más altos en los Andinos. Sin embargo, en cuanto a desempeño económico, los países andinos casi duplican a los del Cono Sur. En todo caso, en estas dos sub-regiones se tienen con mucha influencia, los pesos desfavorables en los indicadores, de Venezuela y Brasil (Elliot, 2000).

\section{COLOMBIA Y PAÍSES DE LA ALIANZA DEL PACÍFICO 2015 Y 2016: COMPORTAMIENTO ECONÓMICO}

De conformidad con cifras dadas a conocer por la Comisión Económica para América Latina y el Caribe (CEPAL, 2015) las perspectivas de crecimiento económico para finalizar este año, confirman, en términos generales, el impacto que ha tenido el fin de la era de altos precios de materias primas de exportación regionales -2003 a 2014.

No obstante, tal y como puede apreciarse en el Cuadro 5, Perú mantiene un notable desempeño, el mejor del grupo. Con esto se ratifica que Costa Rica, Panamá, Perú y Uruguay, son los países que han mantenido un buen desempeño, en función de los datos que se evidencian en Latinoamérica.

Tabla 5. Perú y Alianza del Pacífico 2016: perspectivas de desempeño macroeconómico

\begin{tabular}{lccc}
\hline \multicolumn{1}{c}{ País } & $\begin{array}{c}\text { Crecimiento Económico } \\
\text { (variación \% de PIB) }\end{array}$ & $\begin{array}{c}\text { Inflación } \\
(\%)\end{array}$ & $\begin{array}{c}\text { Desempleo Abierto } \\
\text { (\%) }\end{array}$ \\
\hline Chile & 1.5 & 4.1 & 6.8 \\
Colombia & 2.5 & 7.3 & 9.8 \\
\hline México & 2.4 & 2.9 & 4.1 \\
Perú & 3.7 & 3.1 & 6.0 \\
\hline
\end{tabular}

Fuente: Comisión Económica para América Latina y el Caribe (CEPAL) (2015) Balance Preliminar de las Economías de América Latina y el Caribe 2015. (Santiago de Chile, Chile: CEPAL); y estadísticas del Fondo Monetario Internacional (véase: http://www.imf. org/external/index.htm). 
Los índices de inflación y de desempleo son más preocupantes en el caso de Colombia. Nótese que es el país del grupo que tiene estos indicadores en niveles altos. A esto se agrega que la Dirección de Administración Nacional de Estadística (DANE) en 2012 cambió los indicadores para medir la desocupación abierta en el país. Se estima que si se hubiesen conservado las metodologías anteriores, la cifra de desempleo abierto en Colombia tendría valores más altos.

En todo caso, los desafíos de la ocupación en Latinoamérica, se centran en el subempleo o prevalencia de economías informales. La estimación de empleo para Perú es relativamente baja, pero es de advertir que estas mediciones se hacen en el área de las ciudades principales del país.

En la tabla 6 puede observarse, especialmente en el caso de Perú, cómo la caída del crecimiento económico de los países de la AP se ve asociada a la caída de los precios internacionales de las exportaciones. Durante el período 2003 a 2014 el crecimiento de ingreso por exportaciones en los países latinoamericanos se debió fundamentalmente a la factura, al precio de los productos en los mercados foráneos, más que al quantum o cantidad de las exportaciones (CEPAL, 2015).

Tabla 6. Perú y Alianza del Pacífico 2010-2015: crecimiento económico (Tasas anuales de variación del producto interno bruto)

\begin{tabular}{lcccccc}
\hline \multicolumn{1}{c}{ País } & $\mathbf{2 0 1 0}$ & $\mathbf{2 0 1 1}$ & $\mathbf{2 0 1 2}$ & $\mathbf{2 0 1 3}$ & $\mathbf{2 0 1 4}$ & $\mathbf{2 0 1 5}$ \\
\hline Chile & 5.8 & 5.7 & 5.5 & 4.2 & 1.9 & 2.1 \\
Colombia & 4.0 & 6.6 & 4.1 & 4.9 & 4.6 & 3.1 \\
México & 5.2 & 3.9 & 4.0 & 1.4 & 2.2 & 2.5 \\
Perú & 8.3 & 6.3 & 6.1 & 5.9 & 2.4 & 2.8 \\
\hline
\end{tabular}

Fuente: Comisión Económica para América Latina y el Caribe (CEPAL) (2015) Balance Preliminar de las Economías de América Latina y el Caribe 2015. (Santiago de Chile, Chile: CEPAL); y The Economist Intelligence Unit (http://www.eiu.com/home.aspx).

De manera más pormenorizada, la tabla 7, muestra el crecimiento económico de los países miembros de la AP en función de sectores productivos. Para Chile y Colombia, los sectores agropecuarios tienden a contraerse. En el caso colombiano la situación se agrava al detallarse que la industria también tiende a mostrar un nivel negativo de crecimiento.

Estos serían factores que estarían asociados a los altos niveles de desempleo que se observan en Colombia. La economía de este país, por otra parte, muestra niveles de aumento aceptables, pero los sectores que son más dinámicos han sido la minería, líneas productivas relacionadas con energía y el sector financiero. Se trata de sectores que no tienen mayor efecto multiplicador en el empleo.

Los sectores económicos que si generarían mayores niveles de ocupación especialmente para grupos sociales que no tienen mayor preparación laboral, son: (i) infraestructura urbana; (ii) infraestructura rural; (iii) construcción; y (iv) turismo. Son sectores que son privilegiados, en particular con base en inversiones públicas 
y que tienden a abatir altos niveles de desempleo abierto, subempleo y personas vinculadas a la economía informal.

Tabla 7. Perú y Alianza del Pacífico 2015: crecimiento económico por sectores (\% de variación anual)

\begin{tabular}{|c|c|c|c|c|}
\hline Sector Económico & Chile & Colombia & México & Perú \\
\hline Agropecuario & -0.6 & -2.6 & 4.3 & 3.8 \\
\hline Industrial & 2.6 & -0.7 & 13.6 & 5.6 \\
\hline Transporte & 4.2 & 4.1 & No disponible & No disponible \\
\hline Construcción & 8.4 & 3.6 & 17.4 & 3.4 \\
\hline Comercio & 8.1 & 4.1 & 9.7 & 8.8 \\
\hline Minería e Energía & 4.3 & 5.9 & -0.1 & -0.2 \\
\hline
\end{tabular}

Fuente: Comisión Económica para América Latina y el Caribe (CEPAL) (2015) Balance Preliminar de las Economías de América Latina y el Caribe 2015. (Santiago de Chile, Chile: CEPAL); y estadísticas del Fondo Monetario Internacional (véase: http://www.imf. org/external/index.htm).

\section{COLOMBIA Y PAÍSES DE LA ALIANZA DEL PACÍFICO: INFLACIÓN Y VARIABLES DE EMPLEO}

En la tabla 8 muestra los datos de inflación, y conforme al mismo, los niveles de aumento en el índice de precios al consumidor se evidencian muy controlados en México y Perú. Los valores de este indicador en el caso de Colombia son preocupantes.

Tal y como es normal en economías que tienen la estructura de las latinoamericanas, una apreciación de moneda local, tiende a promover con mayor intensidad las importaciones, al abaratar la moneda de referencia, en este caso el dólar estadounidense. De manera paralela lo más esperado es que con la apreciación monetaria se tiende a desestimular las exportaciones. Estos dos factores redundan en lanzar en niveles deficitarios la balanza comercial.

Por el contrario, cuando la moneda tiende a la devaluación o depreciación, la tendencia consistiría en promover más las exportaciones y a provocar un descenso en las importaciones. Esto último, debido a que la moneda dura o de referencia se hace más cara.

Sin embargo, dado que la estructura de importación de los países latinoamericanos se refiere a insumos, equipo y en general bienes intermedios de producción, lo que ocurre es que la devaluación encarece componentes productivos que son importados. El resultado es que fácilmente se importa inflación, dado que el nivel de precios tiende generalizadamente a ser mayor. Ese es el vínculo entre la política cambiaria, la inflación y la capacidad agregada de compra que se tiene en los mercados internos. 
Tabla 8. Perú y Alianza del Pacífico 2013-2015: inflación

(Tasa de variación en \% anual al final de cada período)

\begin{tabular}{lccc}
\hline \multicolumn{1}{c}{ País } & $\mathbf{2 0 1 3}$ & $\mathbf{2 0 1 4}$ & $\mathbf{2 0 1 5}$ \\
\hline Chile & 3.1 & 4.6 & 4.0 \\
Colombia & 1.9 & 3.7 & 5.9 \\
México & 4.0 & 4.1 & 2.5 \\
Perú & 2.9 & 3.2 & 3.7 \\
\hline
\end{tabular}

Fuente: Comisión Económica para América Latina y el Caribe (CEPAL) (2015) Balance Preliminar de las Economías de América Latina y el Caribe 2015. (Santiago de Chile, Chile: CEPAL).

En términos de desempleo, tal y como fue expuesto el caso más preocupante es el de Colombia. Con relativamente alto desempleo y altos niveles de inflación, el país tiene el riesgo de que se presente el fenómeno de estanflación. Esto contribuye a mantener o bien incrementar, en el peor de los casos, los niveles de personas viviendo en condiciones de pobreza o indigencia (CEPAL, 2010).

Los resultados del desempleo abierto están relacionados con los niveles de la economía informal o subterránea, esto es, la influencia del sub-empleo. La desocupación tiende a aumentar en Chile y en Perú, aunque con niveles que parecen estar controlados o estabilizados. Una de las discrepancias o diferencias en términos de la política económica entre Latinoamérica y el Sud-Este Asiático es que mientras en la primera de las mencionadas se privilegia la estabilidad -de allí la función más que prioritaria de los bancos centrales a controlar la inflación- para las economías asiáticas, lo más importante es el crecimiento.

Es cierto que la tendencia es que a mayor crecimiento también aumentan las posibilidades de inflación, pero sin crecimiento, aunque con estabilidad, la creación de empleo no se dinamiza lo suficiente y los mercados internos tienden a no ampliarse.

Tabla 9. Colombia y Alianza del Pacífico 2013-2015: desempleo abierto (Tasas anuales en porcentaje)

\begin{tabular}{lrll}
\hline País & $\mathbf{2 0 1 3}$ & $\mathbf{2 0 1 4}$ & $\mathbf{2 0 1 5}$ \\
\hline Chile $^{1}$ & 5.9 & 6.4 & 6.3 \\
Colombia $^{2}$ & 10.6 & 9.9 & 9.6 \\
México $^{3}$ & 5.7 & 5.8 & 5.1 \\
Perú $^{4}$ & 5.9 & 6.0 & 6.5 \\
\hline
\end{tabular}

Notas: 1/ Chile, total nacional; 2/ Colombia, 13 principales ciudades; 3/ México, áreas urbanas; 4/ Perú, Lima metropolitana.

Fuente: Comisión Económica para América Latina y el Caribe (CEPAL) (2015) Balance Preliminar de las Economías de América Latina y el Caribe 2015. (Santiago de Chile, Chile: CEPAL). 


\section{COLOMBIA Y PAÍSES DE LA ALIANZA DEL PACÍFICO: INVERSIÓN EN ECONOMÍA REAL}

Se reconoce que existen dos grandes sectores económicos que actúan conjuntamente, pero que se excluyen en cuanto a la captación de dinero. Por una parte está el sector real. Es el sector que más importa para fines de promoción y consolidación del desarrollo, dado que tiene relación directa con la creación de empleo y la generación de bienes y servicios.

Por otra parte está el sector financiarista de la economía. Es el sector cuyas rentas se obtienen a partir de las transacciones bursátiles, de procesos de compraventa de acciones, bonos y en general derivados financieros. Este sector no genera de manera estricta, una producción de bienes y servicios, sino que la transacción se produce a partir de papeles o títulos (CEPAL, 2009).

Con base en lo anterior, lo más importante en función de ampliar las capacidades de las personas y de aumentar las oportunidades para las mismas, es la economía real. Por ello esta sección se centra en la caracterización de la formación de capital fijo y en la inversión extranjera directa.

Tabla 10. Perú y Alianza del Pacífico 2010-2015: formación bruta de capital fijo (Como \% del PIB)

\begin{tabular}{lcccccc}
\hline País & 2010 & 2011 & 2012 & 2013 & 2014 & 2015 \\
\hline Chile & 21 & 23 & 24 & 24 & 22 & 22 \\
Colombia & 21 & 22 & 25 & 24 & 26 & 26 \\
México & 21 & 22 & 22 & 22 & 22 & 21 \\
Perú & 24 & 25 & 26 & 26 & 25 & 25 \\
\hline
\end{tabular}

Fuente: Comisión Económica para América Latina y el Caribe (CEPAL) (2015) Balance Preliminar de las Economías de América Latina y el Caribe 2015. (Santiago de Chile, Chile: CEPAL).

En la tabla 10, la información sobre formación de capital fijo en los países de la Alianza para el Pacífico, muestra una situación relativamente estable en esas naciones, con rangos de inversión en función del producto interno bruto (PIB) que van de 21 a 26 por ciento. Perú es el país que mantiene, en promedio, índices mayores, con diferencias matemáticas, más no significativas con las cifras de las otras naciones.

El indicador de inversión extranjera directa se relaciona con la credibilidad y de las instituciones y las condiciones económicas y socio-políticas que presenta un país. La confianza que se requiere para atraer capitales productivos especialmente en la economía real, se vincula a seis factores fundamentales: (i) estabilidad política; (ii) estabilidad macroeconómica -incluyendo la de tipo cambiario; (iii) infraestructura física; (iv) educación y en general capacitación del capital humano; (v) instituciones y estado de derecho; y (vi) factores culturales. 
Es con base en los anteriores factores, por ejemplo, que los capitales productivos tienden a buscar lugares como Singapur, en lugar de países que tienen mucha debilidad institucional e infraestructura incipiente tal el caso de Haití. Aunque este último país, asolado por tragedias (la última de ellas por el terremoto del 12 de enero de 2010) tiene mano de obra substancialmente más barata.

Tabla 11. Colombia y Alianza del Pacífico 2012-2014: inversión extranjera directa (Millones de US\$)

\begin{tabular}{lrrr}
\hline \multicolumn{1}{c}{ País } & 2012 & 2013 & \multicolumn{1}{c}{2014} \\
\hline Chile & 7902 & 8956 & 9950 \\
Colombia & 15646 & 8557 & 12252 \\
México & -2739 & 32032 & 16837 \\
Perú & 11840 & 9161 & 7789 \\
\hline
\end{tabular}

Fuente: Comisión Económica para América Latina y el Caribe (CEPAL) (2015) Balance Preliminar de las Economías de América Latina y el Caribe 2015. (Santiago de Chile, Chile: CEPAL).

Los países de la Alianza del Pacífico se muestran consistentes en la atracción de inversión extranjera directa. La única cifra negativa corresponde a México en 2012. Uno de los factores que puede explicar esta situación es el impacto de la crisis financiera que tuvo su punto de inflexión en septiembre de 2008 con la quiebra del banco Lehman Brothers.

Como se sabe la economía mexicana se encuentra íntimamente ligada a la economía estadounidense que se constituye en mercado natural del país latinoamericano y de toda la sub-región de Centroamérica y el Caribe. Cerca de un 87 por ciento de las exportaciones mexicanas tienen por destino la potencia del norte.

\section{COLOMBIA Y PAÍSES DE LA ALIANZA DEL PACÍFICO: DEUDA EXTERNA}

La variable de deuda externa se presenta de manera conjunta con la transferencia neta de recursos que reciben los países por dos razones fundamentales. En primer lugar, la deuda externa tiende a asociarse con la vulnerabilidad económica externa que tiene un país. Especialmente las naciones latinoamericanas vieron cómo, a partir de agosto de 1982, se desataba el problema de deuda externa que provocó -entre otros desenlaces- la aplicación de programas de ajuste macroeconómico.

Con ello, a fin de cancelar las deudas especialmente con entidades privadas -el Club de París- apoyó medidas que restringieron el poder de las instituciones, con lo cual las oportunidades vía el empleo, se vieron disminuidas, se disminuyó la capacidad institucional que no era de muy grande cobertura, y se aumentaron los niveles de atención social para muchos grupos de la región (Graham, 2002). 
Tabla 12. Colombia y Alianza del Pacífico 2013-2015: Deuda externa bruta (Millones de US\$ al final de cada período)

\begin{tabular}{lrrr}
\hline \multicolumn{1}{c}{ País } & $\mathbf{2 0 1 2}$ & $\mathbf{2 0 1 3}$ & \multicolumn{1}{c}{2014} \\
\hline Chile & 132632 & 145666 & 148716 \\
Colombia & 91923 & 101212 & 108199 \\
México & 254793 & 282479 & 291029 \\
Perú & 60823 & 64512 & 63789 \\
\hline
\end{tabular}

Fuente: Comisión Económica para América Latina y el Caribe (CEPAL) (2015) Balance Preliminar de las Economías de América Latina y el Caribe 2015. (Santiago de Chile, Chile: CEPAL).

En segundo lugar, el problema finalmente no es tener deuda que puede llegar a ser un gran peso para la economía de un país -en especial se le caracteriza como porcentaje del total de la economía- como el peso que tiene en el presupuesto al pasar del déficit primario al secundario de un país, y como deuda per cápita.

El problema de la deuda es no sólo su destino -si es para egresos o gastos operativos o de inversión- o bien si está destinada para inversiones productivas en el país. Un ejemplo de esto último es el caso de la ampliación del Canal de Panamá, obra que requirió cerca de 10 años de trabajo, donde se invirtieron unos 5,200 millones de dólares, y que fue inaugurada el domingo 26 de junio de 2016 .

Esta obra es indiscutiblemente una obra estratégica que promueve el crecimiento, el desarrollo económico del país y que permite a esta economía una inserción más competitiva en el ámbito internacional. De conformidad con lo expuesto en el Cuadro 12, la deuda externa tiende a mantenerse relativamente estable en los países de la AP, aunque tiende a subir de manera relativamente significativa en el caso de Colombia. Es de recordar que este país, junto a República Dominicana y Guatemala, fueron los tres casos de “escape a la trampa de la deuda externa” de los años setenta.

En ese tiempo, mucho países, evitando llevar a cabo un ajuste macroeconómico producto de las alzas del petróleo -1973 y 1979- adquirieron notables montos de deuda externa.

\section{COLOMBIA Y PAÍSES DE LA ALIANZA DEL PACÍFICO: INDICADORES DE POLÍTICA FISCAL}

En general, las condiciones de la política fiscal determinan la capacidad institucional en los diferentes países. En América Latina, más que ser instituciones que propenden a la inclusión social -tal y como lo sostiene Acemoglu y Robinson (2012) en muchos casos en Latinoamérica, las entidades públicas padecen del problema de la corrupción, son cooptadas por sectores que logran rentas extraordinarias y no permiten -tanto como debieran- la formación de mercados incluyentes. 
Tabla 13. Alianza del Pacífico 2014-2015: Ingresos de los gobiernos centrales (Como \% del PIB)

\begin{tabular}{llccc}
\multicolumn{1}{c}{ País } & & Ingreso Total & \multicolumn{2}{c}{ Ingresos Tributarios } \\
\hline Chile & 2014 & $\mathbf{2 0 1 5}$ & $\mathbf{2 0 1 4}$ & $\mathbf{2 0 1 5}$ \\
\hline Colombia & 20.7 & 20.5 & 18.1 & 18.5 \\
\hline México & 16.7 & 16.3 & 14.3 & 14.5 \\
Perú & 23.3 & 22.1 & 10.6 & 10.6 \\
\hline
\end{tabular}

Fuente: Comisión Económica para América Latina y el Caribe (CEPAL) (2015) Balance Preliminar de las Economías de América Latina y el Caribe 2015. (Santiago de Chile, Chile: CEPAL).

La situación antes descrita, no obstante tiene matices, y las condiciones fiscales que se muestran más favorables a la institucionalidad se hacen evidentes en los casos de Uruguay, Costa Rica, Chile, Jamaica y Brasil. Este último país, aún teniendo que enfrentar casos inéditos de crisis política debidos -entre otros factores- a la corrupción de empresas estatales, i.e. Petrobras.

En la tabla 13 se da a conocer el ingreso de los gobiernos en función del total de producción de los diferentes países. Nótese que en ninguno de los casos se supera, excepto por Chile el 15 por ciento de ingresos tributarios. Este dato de Chile, no obstante, es bajo cuando incluso se compara con Estados Unidos, el país que tiene el más bajo indicador de este tipo entre las naciones desarrolladas, con un 32 por ciento de ingresos tributarios en función del producto interno bruto. Los egresos de los gobiernos se dividen en operativos y de inversión, esa es una clasificación. Su análisis completo, escapa a los alcances de este estudio.

\section{COLOMBIA Y PAÍSES DE LA ALIANZA DEL PACÍFICO: INDICADORES DE POLÍTICA MONETARIA}

En términos de la política monetaria, los bancos centrales tienen tres instrumentos a su disponibilidad: (i) movimiento de las tasas de interés; (ii) determinación de los niveles de encaje bancario; y (iii) las operaciones de mercado abierto.

Tabla 14. Colombia y Alianza del Pacífico 2013-2015: tasas de interés de la política monetaria (\%)

\begin{tabular}{lccc}
\hline País & $\mathbf{2 0 1 3}$ & $\mathbf{2 0 1 4}$ & $\mathbf{2 0 1 5}$ \\
\hline Chile & 4.9 & 3.7 & 3.0 \\
Colombia & 3.4 & 3.9 & 4.7 \\
\hline México & 4.0 & 3.2 & 3.0 \\
Perú & 4.2 & 3.8 & 3.3 \\
\hline
\end{tabular}

Fuente: Comisión Económica para América Latina y el Caribe (CEPAL) (2015) Balance Preliminar de las Economías de América Latina y el Caribe 2015. (Santiago de Chile, Chile: CEPAL). 
Por otra parte, los bancos centrales en los países latinoamericanos tienen casi el exclusivo fin de velar por la estabilidad de precios, esto es de evitar el descontrol de la inflación. Algo que dejó dolorosos recuerdos de la "década perdida" de los ochenta, cuando inflaciones galopantes se hicieron presentes en la mayor parte de países de la región.

Con base en el manejo de los tres instrumentos de política monetaria, los bancos centrales tratan de aumentar o disminuir la masa monetaria existente en el sistema de mercado. Al elevar, la idea es que se abarata el crédito y con ello se desalienta a quienes -en lugar de invertir en la economía real- hacen depósitos en los bancos.

De esa manera, de forma simultánea, se tiene mayor disponibilidad de circulante para las inversiones en empresas que producen bienes y servicios, y además promueven la creación de empleo, es decir, se fortalece el emprendimiento. La razón para esto reside en que generalmente a mayor tasa de crecimiento económico, se tiene mayor riesgo de que se desborde un aumento generalizado de precios, es decir que exista inflación. Por ello, con el fin de detener una economía sobrecalentada, se pueden implementar procesos contractivos tanto en la política fiscal como en la monetaria (Reyes, 2008).

Nótese cómo Colombia, no obstante tener una relativamente alta tasa de desempleo, aumenta la tasa de interés del Banco Central. Lo que trata de evitar con ello es un aumento inflacionario, pero a la vez contrae la economía y no permite ampliar la creación de oportunidades de trabajo.

\section{COLOMBIA Y PAÍSES DE LA ALIANZA DEL PACÍFICO: INDICADORES DE POLÍTICA CAMBIARIA}

Tal y como fue expuesto, una política cambiaria que tienda a la apreciación de la moneda lo que produce como tendencia es a hacer menos competitivos los productos de exportación y abarata las importaciones. Con ello se golpea la creación de puestos de trabajo y se tiende a hundir en números rojos o deficitarios, las balanzas comerciales.

La situación contraria debe ocurrir al devaluarse o depreciarse las monedas. Debe reconocerse en esto que una devaluación es producto de una medida deliberada de las autoridades de gobierno, mientras que una depreciación corresponde a los juegos de oferta y demanda monetarios.

Existen casos en los países latinoamericanos en los cuales la devaluación no promueve mayores niveles de exportación. Una causa para ello es la existencia de mercados cautivos, en los cuales se colocan los productos exportables. Este es el caso del petróleo venezolano. Para esta economía, las exportaciones petroleras son cerca de un 90 por ciento del portafolio de bienes y servicios en el exterior. 
Tabla 15. Colombia y Alianza del Pacífico 2012-2015: tipos de cambio (Índice $2005=100$ )

\begin{tabular}{lrrrr}
\hline \multicolumn{1}{c}{ País } & $\mathbf{2 0 1 2}$ & $\mathbf{2 0 1 4}$ & $\mathbf{2 0 1 3}$ & $\mathbf{2 0 1 5}$ \\
\hline Chile & 88.6 & 90.4 & 100.4 & 103.4 \\
Colombia & 72.7 & 76.7 & 80.8 & 99.0 \\
México & 110.6 & 105.1 & 106.6 & 119.8 \\
Perú & 85.7 & 86.5 & 88.7 & 90.5 \\
\hline
\end{tabular}

Fuente: Comisión Económica para América Latina y el Caribe (CEPAL) (2015) Balance Preliminar de las Economías de América Latina y el Caribe 2015. (Santiago de Chile, Chile: CEPAL).

Otra de las razones por las cuales la devaluación no asegura ni mucho menos recuperaciones sostenibles de las exportaciones y de la balanza de pagos, consiste en que las importaciones que se realizan son necesarias para establecer procesos productivos. Con ello, un encarecimiento de las importaciones redunda en un incremento de precios en bienes no transables -que se comercian internamente en los mercados- y con ello, una devaluación puede traducirse en "importación de inflación".

La tabla 15 da a conocer las condicionantes cambiarias de los países de la AP. Los niveles de mayor depreciación se evidencian en México y en Chile. Con menos intensidad en Colombia.

\section{COLOMBIA Y PAÍSES DE LA ALIANZA DEL PACÍFICO: INDICADORES DE COMERCIO EXTERIOR}

Tal y como lo muestra el Cuadro 21 la balanza de bienes y servicios de los países de la Alianza del Pacífico tienen niveles deficitarios desde hace dos años. La situación se explica en función de que las exportaciones han perdido su valor de factura en el medio externo, no obstante los relativos niveles de depreciación monetaria que se han hecho presentes.

Tabla 16. Colombia y Alianza del Pacífico 2014-2015: balanza de bienes y servicios (Millones de US\$)

\begin{tabular}{lcc}
\hline \multicolumn{1}{c}{ País } & $\mathbf{2 0 1 4}$ & $\mathbf{2 0 1 5}$ \\
\hline Chile & -1582 & -4010 \\
Colombia & -2745 & -11268 \\
México & -11893 & -15024 \\
Perú & -3077 & -5361 \\
\hline
\end{tabular}

Fuente: Comisión Económica para América Latina y el Caribe (CEPAL) (2015) Balance Preliminar de las Economías de América Latina y el Caribe 2015. (Santiago de Chile, Chile: CEPAL). 
Aunque México junto a Brasil y Costa Rica son los países que más han logrado diversificar su oferta exportadora, Perú, Chile y Colombia presentan alta dependencia de materias primas en los productos que venden en el exterior. En general uno de los más importantes problemas de la competitividad de América Latina lo constituye la necesidad de fortalecer los niveles de valor agregado en sus exportaciones.

No cumplir con este reto es mantener los escenarios productivos actuales en los cuales se está muy expuesto a los "schock" de la economía internacional, mientras en el mercado doméstico no pueden generar, usualmente, niveles de empleo de gran remuneración.

Es más, conforme ocurre el avance de la tecnología, se constituyen y se consolidan sectores de tecnología de punta -informática, cibernética, biotecnología, satélites y complejos sistemas de computación y nanotecnología- cada vez se requiere de mayores niveles de inversión para poder generar empleos con altas remuneraciones.

Tabla 17. Colombia y Alianza del Pacífico 2014-2015: índices de exportaciones $(2010=100)$

\begin{tabular}{lcccc}
\hline \multicolumn{1}{c}{ País } & \multicolumn{2}{c}{ Valor } & \multicolumn{2}{c}{ Volumen } \\
& $\mathbf{2 0 1 4}$ & $\mathbf{2 0 1 5}$ & $\mathbf{2 0 1 4}$ & $\mathbf{2 0 1 5}$ \\
\hline Chile & 107 & 106 & 110 & 109 \\
Colombia & 139 & 92 & 139 & 128 \\
México & 133 & 128 & 124 & 130 \\
Perú & 110 & 93 & 105 & 103 \\
\hline
\end{tabular}

Fuente: Comisión Económica para América Latina y el Caribe (CEPAL) (2015) Balance Preliminar de las Economías de América Latina y el Caribe 2015. (Santiago de Chile, Chile: CEPAL).

Cuando, por otra parte se comparan los índices de exportaciones, tanto en valores como en volumen o quantum de las mismas, se hace evidente que Colombia y Perú han visto disminuir el valor de sus productos en el extranjero. Esta situación contrasta fundamentalmente con la experiencia de México que ha visto aumentar el valor de los mismos en un 28 por ciento (véase tabla 17).

De nuevo aquí surge la diversificación de oferta exportable de México. Esa diversificación y tener productos que tienen mayor valor agregado, hacen que la economía del país se resguarde de impactos adversos de lo que puede ocurrir en los mercados internacionales. Además, México representa casi un 47 por ciento del total que exporta América Latina, es decir la mitad de la inserción internacional regional por la vía de exportaciones (Dymski, 2010).

En cuanto al volumen de exportación, los cuatro países dan muestras de estar realizando exitosos esfuerzos en este sentido. De nuevo la dificultad estriba en que los productos tienen poco valor agregado y con ello baja la competitividad que se puede desarrollar en la economía internacional. 
Tabla 18. Colombia y Alianza del Pacífico 2014-2015: índices de importaciones

$$
(2010=100)
$$

\begin{tabular}{lcccc}
\hline \multicolumn{1}{c}{ País } & \multicolumn{2}{c}{ Valor } & \multicolumn{2}{c}{ Volumen } \\
& 2014 & $\mathbf{2 0 1 5}$ & $\mathbf{2 0 1 4}$ & $\mathbf{2 0 1 5}$ \\
\hline Chile & 123 & 106 & 116 & 112 \\
Colombia & 160 & 139 & 147 & 140 \\
México & 133 & 133 & 123 & 128 \\
Perú & 141 & 128 & 127 & 128 \\
\hline
\end{tabular}

Fuente: Comisión Económica para América Latina y el Caribe (CEPAL) (2015) Balance Preliminar de las Economías de América Latina y el Caribe 2015. (Santiago de Chile, Chile: CEPAL).

La situación del descenso de los índices en cuanto a valor de exportaciones, contrasta con el aumento del índice del valor de las importaciones. En efecto, tal y como lo muestra la tabla 18, esos valores de productos comprados en el exterior tienden a aumentar. Véase el aumento del 60 por ciento que se tuvo en las importaciones de Colombia en 2014, respecto a los niveles de 2010, aunque ese nivel tendió a mostrar una baja para 2015.

Además de las condiciones, de alza en los índices de valores de importación, véase también cómo fue Colombia, el país que mayor dinamismo mostró en el volumen de importación. Ese índice aumento un 40 por ciento desde 2010 a 2015.

Al tener presente cómo los valores de las exportaciones caen y cómo se elevan los precios de las importaciones, no es de extrañar que los términos de intercambio se debiliten. Esto es lo que se muestra en el Cuadro 19. Es decir que los países latinoamericanos y en general en desarrollo compran cada vez con tendencias alcistas en los precios mientras sus productos se estacan o valen menos.

Todo esto es una tendencia más bien a largo plazo, debido a que existen coyunturas en las cuales los precios de las exportaciones latinoamericanas si pueden tener alzas, tal y como se tuvo durante el período 2003 a 2014.

Tabla 19. Colombia y Alianza del Pacífico 2012-2015: términos de intercambio $(2010=100)$

\begin{tabular}{lcccc}
\hline País & 2012 & 2014 & 2013 & 2015 \\
\hline Chile & 95 & 91 & 93 & 88 \\
Colombia & 108 & 92 & 100 & 72 \\
México & 105 & 99 & 104 & 95 \\
Perú & 105 & 94 & 99 & 89 \\
\hline
\end{tabular}

Fuente: Comisión Económica para América Latina y el Caribe (CEPAL) (2015) Balance Preliminar de las Economías de América Latina y el Caribe 2015. (Santiago de Chile, Chile: CEPAL). 
Otro período de notables alzas ocurrió durante los años setenta con las alzas de petróleo -desfavoreciendo a los importadores de crudo. Y un período de alzas más se tuvo al finalizar la II Guerra Mundial. Esto último favoreció a naciones como Argentina, Colombia y Venezuela, al promoverles mayores niveles de liquidez en divisas en el contexto de su inclusión en los mercados externos.

\section{CONCLUSIONES Y CONSIDERACIONES FINALES}

Dentro del contexto de la Alianza del Pacífico, la situación política más actualizada de Perú se encamina a conservar las políticas de mercado, en un ambiente de crecimiento económico que trata de fortalecer instituciones y de aminorar la inequidad social existente en términos de la distribución de oportunidades en la población.

Uno de los rasgos que más notoriamente ha sido puesto de manifiesto en los análisis de institucionalidad sobre integración, es que la AP se mantiene sin mayores niveles de burocratización interna. Colombia es el país de la AP que si bien es cierto mantiene importantes tasas de crecimiento económico, resiente tener altos índices relativos de desempleo abierto.

Esta condición de la economía colombiana se debe esencialmente a que los sectores que presentan mayor crecimiento en la economía -banca, finanzas, carbón y en general línea de energéticos- no crean tanto efecto multiplicador en la creación de puestos de trabajo, como si es el caso de la construcción, agricultura, vivienda, infraestructura urbana, infraestructura rural y turismo.

Las políticas monetarias han tendido a la elevación de las tasas internas de interés -con mayor evidencia en Colombia- como un mecanismo que trate de evitar o al menos amortiguar la "importación de inflación”, que se tiene al generarse mecanismos que hacen que el dólar se haya encarecido.

Las condicionantes fiscales son propias de países que tienen en general débil el andamiaje institucional y que los márgenes de mejora de cobertura son amplios; se requerirían de pactos fiscales internos con el fin de asegurar, no sólo un correcto uso de los recursos -lucha contra la corrupción- sino también para evitar dinámicas que generan rentismos lucrativos para empresas, pero con alto costo social.

En términos de política económica, lo que el gobierno colombiano debería enfocar es en promover valor agregado de las exportaciones, además de reforzar el carácter de complementariedad productiva en las exportaciones que se realizan dentro del grupo.

Entre los desafíos que tienen los países miembros de la AP se encuentran: (i) promover mercados incluyentes; (ii) disminución de los índices de pobreza e indigencia aún existentes; (iii) fomento de la competitividad en la inserción externa de mercados; (iv) generación de valor agregado en las exportaciones; (v) promoción de complementariedad productiva para una más efectiva integración comercial; y (vi) aseguramiento en la articulación de marcos jurídicos que permitan viabilizar de manera congruente con la integración, el vínculo con terceros países -entre esos temas se incluiría la de normas de origen, arancel externo común y coordinación de políticas macroeconómicas-. 


\section{REFERENCIAS}

1. ACEMOGLU, D. \& ROBINSON, J. (2012). Why nations fail: Origins of power, poverty and prosperity and Poverty. Editorial Books.

2. ACUERDO DE CARTAGENA (1969). Cartagena - Colombia.

3. ALIANZA DEL PACÍFICO (2012). htt://alianzapacifico.net

4. CEPAL (2015). Estudio Económico de América Latina y el Caribe. Santiago. Chile.

5. CEPAL (2009) Panorama Social de América Latina 2009. Santiago de Chile, Chile.

6. CEPAL (2010) América Latina frente al Espejo: Dimensiones Objetivas y Subjetivas de la Inequidad Social yel Bienestar en la Región. Santiago de Chile.

7. DECLARACIÓN DE LIMA (2011). Lima - Perú.

8. DYMSKI, Gerarld (2010). Development as social inclusion: Reflections on the US Sub-prime crisis. New York: Random.

9. ELLIOT, Donnald (2000). ¿Can Doha Still Deliver on the Development Agenda?. Policy Briefs in International Economics. June, No. PB06-5. Institute for International Economics; Washington, D.C. USA.

10. GRAHAM, Carol (2002) Mobility, Opportunity and Vulnerability: The Dynamics of Poverty and Inequality in a Global Economy, en Journal of Human Development, Vol. 3, No. 1, 2002 (New York: UNDP).

11. MERCOSUR. Mercado Común del Sur (2016).

12. ONU (2006). Objetivos de Desarrollo del Milenio. Nueva York.

13. PROMÉXICO (2015) Negocios Proméxico: The Pacific Alliance Issue. (México, D.F.: Proméxico.

14. REYES, Giovanni (2008). Política Económica, Crecimiento y Desarrollo Humano: Principales Relaciones, en Tendencia, 9 (1), pps. 14-28.

15. REYES, Giovanni (2007). Procesos de Integración en América Latina y El Caribe: Caracterización General y Potencialidad de Nuevos Ejes de Integración en Tendencia, 8 (2), pp. 7-42.

16. REYES, Giovanni (2006). América Latina y El Caribe: Integración e Inserción en los Mercados Internacionales en Nómadas, Revista de Ciencias Sociales, 14 (2) pp. 307-330. 